\title{
As notificações das doenças diarreicas agudas em um município do Pantanal
}

\author{
Notifications of acute diarrheal diseases in a municipality in the Pantanal
}

Notificaciones de enfermedades diarreicas agudas en un municipio del Pantanal

Recebido: 21/04/2021 | Revisado: 30/04/2021 | Aceito: 03/05/2021 | Publicado: 17/05/2021

Barbara Ferraz Bühler
ORCID: https://orcid.org/0000-0002-1730-3222
Secretaria Estadual de Saúde de Mato Grosso, Brasil
E-mail: barbaraferraz04@gmail.com
Sandra Mara Fernandes Bonilha
ORCID: https://orcid.org/0000-0003-2845-6774
Prefeitura Municipal de Cáceres, Brasil
E-mail: musquita_sandra@ @ahoo.com.br
Ernandes Sobreira Oliveira Junior
ORCID: https://orcid.org/0000-0002-6953-6917
Universidade do Estado de Mato Grosso, Brasil
E-mail: ernandes.sobreira@gmail.com

\section{Resumo}

A Doença Diarreica Aguda (DDA) constitui um problema de saúde pública global, com 1,7 bilhão de casos na infância por ano. Sua ocorrência pode estar relacionada à fatores sociais, culturais, nutricionais e ambientais, tais como baixas coberturas de saneamento básico e a dinâmica das águas de uma região. Em situações de intensas chuvas e inundações os casos de diarreia tendem a aumentar. Considerando o exposto, o presente trabalho visa comparar a ocorrência de diarreia em Cáceres-MT numa série temporal (2015, 2017 e 2018 - 2016 foi excluído por ausência de dados) com os índices pluviométricos e fluviométricos. As ocorrências de diarreia foram extraídas do SIVEP-DDA; os dados sobre pluviosidade foram obtidos em tabelas disponíveis no site do Instituto Nacional de Meteorologia e os dados sobre o nível do rio em planilhas alimentadas e disponibilizadas pela Marinha do Brasil/Serviço de Sinalização Náutica do Oeste. Nos anos de 2015, 2017 e 2018 foram registrados 5.686 casos de diarreia em Cáceres-MT. Os resultados demonstraram que o aumento das ocorrências de DDA está significativamente relacionado com o aumento do nível do Rio Paraguai. Durante os meses de águas baixas, as ocorrências de DDA reduzem $28 \%$ em relação ao período de águas altas, porém sem uma relação significativa com a redução do nível do rio. O uso da água não tratada nesta época pode estar contribuindo para o aumento das DDAs, indicando uma relação entre a dinâmica das águas e os casos de diarreia em Cáceres-MT.

Palavras-chave: Doença tropical; Pluviometria; Fluviometria.

\begin{abstract}
Acute Diarrheal Disease (ADD) is a global public health problem, with 1.7 billion childhood cases per year. Its occurrence may be related to social, cultural, nutritional and environmental factors, such as low coverage of basic sanitation and the dynamics of waters in a region. In situations of intense rain and flooding, cases of diarrhea tend to increase. Considering the above, the present study aims to compare the occurrence of diarrhea in Cáceres-MT in a time series (2015, 2017 and 2018 - 2016 was excluded due to lack of data) with the pluviometric and fluviometric indices. The occurrences of diarrhea were extracted from the SIVEP-DDA; the data on rainfall were obtained from tables available on the website of the National Institute of Meteorology and the data on the level of the river in spreadsheets fed and made available by the Brazilian Navy / West Nautical Signaling Service. In 2015, 2017 and 2018, 5,686 cases of diarrhea were recorded in Cáceres-MT. The results showed that the increase in occurrences of ADD is significantly related to the increase in the level of the Paraguay River. During the low water months, the occurrences of ADD reduce $28 \%$ in relation to the high water period, but without a significant relationship with the reduction in the level of the river. The use of untreated water at this time may be contributing to the increase in ADI, indicating a relationship between water dynamics and diarrhea cases in Cáceres-MT.
\end{abstract}

Keywords: Tropical disease; Pluviometry; Fluviometry. 


\begin{abstract}
Resumen
La Enfermedad Diarreica Aguda (EDA) es un problema de salud pública mundial, con 1.700 millones de casos infantiles por año. Su ocurrencia puede estar relacionada con factores sociales, culturales, nutricionales y ambientales, como la baja cobertura de saneamiento básico y la dinámica de las aguas en una región. En situaciones de lluvia intensa e inundaciones, los casos de diarrea tienden a aumentar. Teniendo en cuenta lo anterior, el presente estudio tiene como objetivo comparar la ocurrencia de diarrea en Cáceres-MT en una serie temporal (2015, 2017 y 2018 2016 se excluyó por falta de datos) con los índices pluviométricos y fluviométricos. Las ocurrencias de diarrea se extrajeron del SIVEP-DDA; los datos sobre precipitaciones se obtuvieron de tablas disponibles en el sitio web del Instituto Nacional de Meteorología y los datos sobre el nivel del río en hojas de cálculo alimentadas y puestas a disposición por la Armada de Brasil / Servicio de Señalización Náutica del Oeste. En 2015, 2017 y 2018 se registraron 5.686 casos de diarrea en Cáceres-MT. Los resultados mostraron que el aumento de ocurrencias de ADD está significativamente relacionado con el aumento del nivel del río Paraguay. Durante los meses de aguas bajas, las ocurrencias de ADD se reducen en un $28 \%$ en relación al período de aguas altas, pero sin una relación significativa con la reducción del nivel del río. El uso de agua no tratada en este momento puede estar contribuyendo al aumento de la IDA, lo que indica una relación entre la dinámica del agua y los casos de diarrea en Cáceres-MT.
\end{abstract}

Palabras clave: Enfermedad tropical; Pluviometría; Fluviometría.

\title{
1. Introdução
}

A doença diarreia aguda (DDA) é uma síndrome clínica de diversas etiologias que se caracteriza por alterações do volume, consistência e frequência das fezes, mais frequentemente associada com a liquidez das fezes e o aumento no número de evacuações. Pode estar acompanhada de vômitos, febre, cólicas e dor abdominal. Quando apresenta muco e sangue recebe o nome de disenteria. Em geral é autolimitada, mas, dependendo do grau de desidratação pode levar a morte (São Paulo, 2008). Sua ocorrência, especialmente nos países em desenvolvimento está relacionada a fatores de ordem social, cultural, ambiental e nutricional (Souza, Silva, Loureiro \& Sousa, 2012). Sobretudo nas cidades nas quais a carência de saneamento básico culmina em contaminação ambiental, a transmissão das diarreias pode acontecer por ingestão hídrica ou alimentos mal higienizados (Papini, 2009).

As DDAs são as principais causas de morbimortalidade infantil, constituindo um dos mais graves problemas de saúde pública global, com 1,7 bilhão de casos na infância por ano. Em 2015, foi a segunda principal causa de mortalidade em menores de 5 anos no mundo, contribuindo com $9 \%$ das mortes nessa faixa etária. No Brasil, a diarreia passou da $8^{\text {a causa de }}$ morte, em 1990, para a 30ª em 2010, o que representa redução de 82\%. Em relação à morte prematura, passou do $4^{\circ}$ lugar, em 1990, para o 25, em 2010; de uma maneira geral, houve diminuição das mortes por diarreia para o período de 1990 a 2010. Apesar disso, entre 2007 e 2015, o número de casos de DDA no Brasil tem apresentado comportamento ascendente, com média aproximada de 4 milhões de registros por ano e incremento médio de aproximadamente 186 mil casos ao ano (Brasil, 2018).

Consta em Buhler, Neves, Ignotti \& Souza (2012), que a região Centro-Oeste apresentou a $3^{a}$ maior proporção de doenças diarreicas em menores de 05 anos no Brasil e dados de 2012 apontam que Mato Grosso (MT) e Mato Grosso do Sul (MS) possuem as doenças infecciosas e parasitárias como a $3^{a}$ causa de óbitos infantis, sendo as diarreicas responsáveis por 323 internações em menores de 01 ano de idade nesses estados no ano mencionado.

Com o objetivo de acompanhar as causas das diarreias e detectar alterações no padrão endêmico da doença nos municípios brasileiros, o Ministério da Saúde lançou em 1992, o programa de Monitorização das Doenças Diarreicas Agudas MDDA, cujo fluxo estabelece que os dados de atendimento de DDA sejam enviados das unidades de saúde para a vigilância epidemiológica municipal (Nível Local). Após recebidos em planilhas, os dados são transferidos para o sistema de informação - SIVEP DDA (Brasil, 2010). 
Assim, fundamentado na série histórica (semanal) de DDA registrada junto ao SIVEP, o município pode avaliar se está num patamar esperado de casos ou enfrentando um surto da doença, adotando, seguidamente, as medidas de profilaxia e assistência para evitar novos casos. Ademais, essa avaliação também permite ao município verificar se os casos registrados são de menor gravidade ou se requerem intervenções mais rigorosas, cujo objetivo seria de prevenir um desfecho mais trágico aos pacientes, tal como o óbito.

No município de Cáceres - MT as unidades que atendem as DDAs são àquelas de atenção básica, urgência/emergência e ambulatoriais (em locais em que estão ausentes as estratégias de saúde da família). Relatório produzido em conjunto pela WWF e Instituto Trata Brasil (2011) demonstrou que no ano de 2011 os gastos com tratamento para diarreia em Cáceres foram de aproximadamente R \$30.000,00 (Trinta Mil Reais) - correspondente a 78 internações. Esses valores para um município de baixa arrecadação como Cáceres (levantamento realizado pela FNP - Frente Nacional dos Prefeitos e divulgado por meio do relatório "g100: municípios populosos com baixa receita per capita e alta vulnerabilidade sócio econômica" colocam Cáceres na posição 43 entre 100 municípios pesquisados) podem ser considerados penosos. Neste sentido, se as ações de prevenção/intervenção acontecessem de maneira mais sistematizada, vários casos de DDAs poderiam ser evitados, abrindo possibilidades para investimento em outras áreas, inclusive em ações/estruturas voltadas para a saúde pública.

Diante do exposto, considerando a importância das DDAs para a morbimortalidade, especialmente em crianças; considerando que a prevenção da ocorrência de DDAs depende do registro de informação que subsidie intervenções adequadas; considerando que a vigilância da DDA em Cáceres necessita de melhorias em termos quanti-qualitativos, propõe-se no presente trabalho descrever aspectos relacionados à notificação das diarreias no município de Cáceres entre os anos de 2014 e 2018.

\section{Métodos}

O estudo ora apresentado foi realizado no município de Cáceres - MT, cujo número de habitantes está estimado em 94.376 - zona urbana e rural (IBGE - 2019). Possui em seu território 13 ESFs (Estratégias de Saúde da Família) o que lhe confere uma cobertura de $38,00 \%$. Se somadas as unidades ambulatoriais (postos de saúde sem ESF e ambulatórios da criança e da mulher) a cobertura de atenção básica soma 58,51\%. O índice de desenvolvimento humano é 0,708, conforme IBGE (2010).

Uma de suas principais atividades econômicas é a pecuária, com um dos maiores rebanhos bovinos do Brasil (IBGE, 2010); o setor terciário (comércio e serviços - educação e saúde) representa para a economia local 97,00\% das empresas constituídas - públicas e privadas (Mato Grosso, 2007). O turismo regional, que tem no Rio Paraguai uma porta de entrada para o Pantanal e na atividade pesqueira o seu maior enfoque, está concretizado como uma alternativa de geração de divisas.

Ao lado de Cuiabá-MT, Rondonópolis-MT, Várzea Grande-MT e Corumbá-MS, Cáceres constitui um dos principais centros populacionais da rede hidrográfica do Paraguai (ANA, 2010).

Atualmente, segundo dados do SISAGUA (2019), 98,56\% da população cacerense é contemplada pelo sistema oficial de abastecimento de água. Os mananciais utilizados para abastecimento são o Rio Paraguai (área urbana - predominantemente) e poços tubulares profundos (zona rural). De acordo com o SIVEP - DDA, existem em 2019, 18 unidades atendendo as diarreias em abrangência municipal. Acrescenta-se que o município é cortado por 4 córregos principais que são reconhecidamente utilizados para o despejo clandestino de esgoto (Oliveira Junior et al, 2014; Oliveira Junior et al 2020).

O presente pesquisa classifica-se como descritiva, de natureza quantitativa (Gil, 2008) e foi desenvolvida a partir de dados de sistemas de informação/sites oficiais (citados abaixo) e planilhas de notificações das diarreias no município de Cáceres entre os anos de 2014 a 2018. A população referida no estudo é aquela cujos dados de notificação por DDA constam 
em registros do SIVEP-DDA e arquivos internos (formulários) da vigilância epidemiológica (VE) municipal. Casos com sintomatologia diarreica atendidos em unidade de saúde sentinela (US) para a monitorização de doenças diarreicas agudas (MDDA) no município de Cáceres e registrados no SIVEP-DDA, em base municipal, no período de 2014 a 2018 foram utilizados para a análise.

Para analisar o comportamento das diarreias no município de Cáceres entre os anos de 2014 a 2018 foram utilizados os dados (total de diarreias, faixa etária de acometimento da diarreia, plano de tratamento adotado, total de unidades notificadoras, percentual de unidades repassando informação semanal) registrados junto ao SIVEP DDA.

Para calcular o percentual de envio de informações anualmente, o total de unidades notificadoras cadastradas em cada ano foi multiplicado pelo total de semanas a informar, naquele período correspondente. O resultado encontrado foi comparado (em regra de três simples) com o registro de semanas informadas no SIVEP DDA (excluindo-se as semanas silenciosas) multiplicado, igualmente, pelo número de unidades cadastradas, obtendo-se ao final, a proporção de envio de informação em cada ano da série histórica.

Para identificar quais unidades enviaram os dados semanais regularmente, bem como espacializar as diarreias por localidade (bairro e/ou comunidade rurais) em Cáceres, foi selecionado o ano de 2018, por ser o mais recente da série histórica; os dados espacializados foram aqueles notificados pela Unidade de Pronto Atendimento Municipal - PAM; a escolha dessa unidade para análise das DDAs por localidade baseou-se nos seguintes critérios: 1) envio regular das planilhas (100\% em 2018); 2) por ser unidade de pronto atendimento pode contemplar usuários de todas as localidades (urbanas e rurais) do município. O levantamento dessas informações foi realizado a partir da leitura das planilhas de DDA arquivadas pela VE municipal. A população utilizada para o cálculo de incidência (geral) das diarreias nos anos de 2014 a 2018 foi extraída do site DATASUS (tabnet.datasus.gov.br).

Para cálculo da incidência por faixa etária foram utilizados dados de população disponibilizados pelo IBGE; por estarem disponibilizados pelo site apenas os dados populacionais de 2014 e 2015, o valor para os outros anos 2016,2017 e 2018 foi estimado pela média dos anos anteriores (2014 e 2015). Considerando as diferenças de faixas etárias populacionais disponibilizadas pelo IBGE e aquelas utilizadas pelo SIVEP DDA, fez-se necessário um ajuste, conforme Quadro 1:

Quadro 1 - Demonstrativo de valores populacionais utilizados para cálculo de diarreia em crianças.

\begin{tabular}{|c|c|l|}
\hline Faixa Disponível no IBGE & Faixa utilizada pelo SIVEP DDA & $\begin{array}{l}\text { Utilizada para o cálculo de } \\
\text { incidência }\end{array}$ \\
\hline 0 a 4 anos & Menor de 1 ano/1 a 4 anos & $\begin{array}{l}\text { Foram somados total de } \\
\text { casos de diarreia em crianças } \\
\text { menores de } 1 \text { ano e total em } \\
\text { crianças de } 1 \text { a } 4 \text { anos; o resultado } \\
\text { foi dividido pela pop de } 0 \text { a } 4 \text { anos }\end{array}$ \\
\hline
\end{tabular}

Fonte: IBGE e SIVEP/DDA.

Os dados de pluviosidade foram obtidos em tabelas disponíveis no site do Instituto Nacional de Meteorologia INMET e os dados sobre o nível do rio em planilhas alimentadas e disponibilizadas pela Marinha do Brasil/Serviço de Sinalização Náutica do Oeste. Todos os dados foram analisados por meio de excel - Microsoft Office®/2016. Os aspectos éticos relacionados à coleta e análise dos dados deste trabalho, estão estabelecidos pela Resolução $\mathrm{N}^{\circ} 510$, de 07 de abril de 2016 do Conselho Nacional de Saúde (CNS). 


\section{Resultados}

Tabela 1 - Número de casos de diarreia entre os anos de 2014 e 2018 no município de Cáceres - MT.

\begin{tabular}{ll}
\hline Ano & Total de Casos \\
2014 & 2.575 \\
2015 & 2.334 \\
2016 & 1.096 \\
2017 & 1.589 \\
2018 & 1.763 \\
\hline TOTAL & $\mathbf{9 . 3 5 7}$ \\
\hline
\end{tabular}

Fonte: Autores. Dados disponibilizados pela SIVEP/DDA Cáceres (consulta em 19.07.2019).

No período de 2014 a 2018 foram registrados 9.357 casos de diarreia junto ao SIVEP DDA do município de Cáceres, de acordo com os valores apresentados na Tabela 1.

Figura 1 - Incidência de diarreia entre os anos de 2014 e 2018, Município de Cáceres/MT (n=9.357).
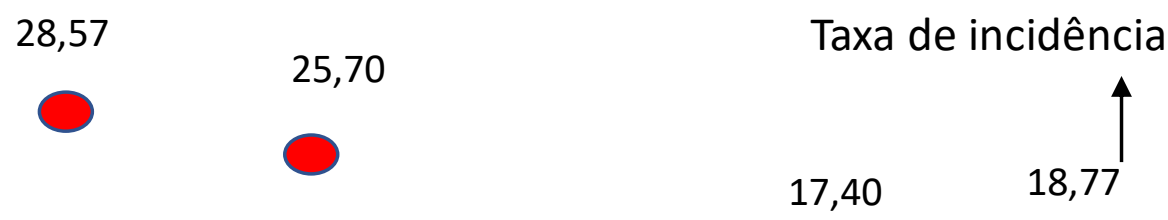

12,05

Fonte: Autores. Dados disponibilizados pela SIVEP/DDA - Cáceres. Consulta em 19.07.2019.

A maior incidência e também o maior número de casos foram registrados no ano de 2014. A menor incidência, acompanhada do menor número de casos, ficou para o ano de 2016 (Figura 1).

Tabela 2 - Proporção de Unidades repassando informação para a VE Municipal entre os anos de 2014 a 2018 no município de Cáceres/MT. Dados em negrito demonstram as maiores e menores proporções.

\begin{tabular}{ccccc}
\hline Ano & $\begin{array}{l}\text { Total de } \\
\text { com } \\
\text { implantado (anual) }\end{array}$ & $\begin{array}{c}\text { Unidades } \\
\text { MDDA }\end{array}$ & $\begin{array}{l}\text { Total de Unidades que } \\
\text { informou (anual) }\end{array}$ & $\begin{array}{l}\text { Proporção de Unidades } \\
\text { repassando } \\
\text { informação para VE (\%) }\end{array}$ \\
\hline $\mathbf{2 0 1 4}$ & 624 & 552 & 88,46 \\
$\mathbf{2 0 1 5}$ & 624 & 576 & $\mathbf{9 2 , 3 0}$ \\
$\mathbf{2 0 1 6}$ & 624 & 396 & 63,46 \\
$\mathbf{2 0 1 7}$ & 648 & 535 & 82,56 \\
\hline
\end{tabular}

Fonte: Autores. Dados disponibilizados pela SIVEP/DDA Cáceres (Consulta em 04.08.2019). 
Research, Society and Development, v. 10, n. 5, e57910515314, 2021

(CC BY 4.0) | ISSN 2525-3409 | DOI: http://dx.doi.org/10.33448/rsd-v10i5.15314

O percentual de unidades notificadoras de diarreia que repassaram informação para vigilância epidemiológica (VE) municipal no período estudado consta na Tabela 2:

O ano com melhor proporção de envio de informação foi 2015, e a mais reduzida proporção foi identificada em 2018.

Figura 2 - Incidência de Diarreia por faixa etária nos anos de 2014 e 2018 no município de Cáceres/MT.

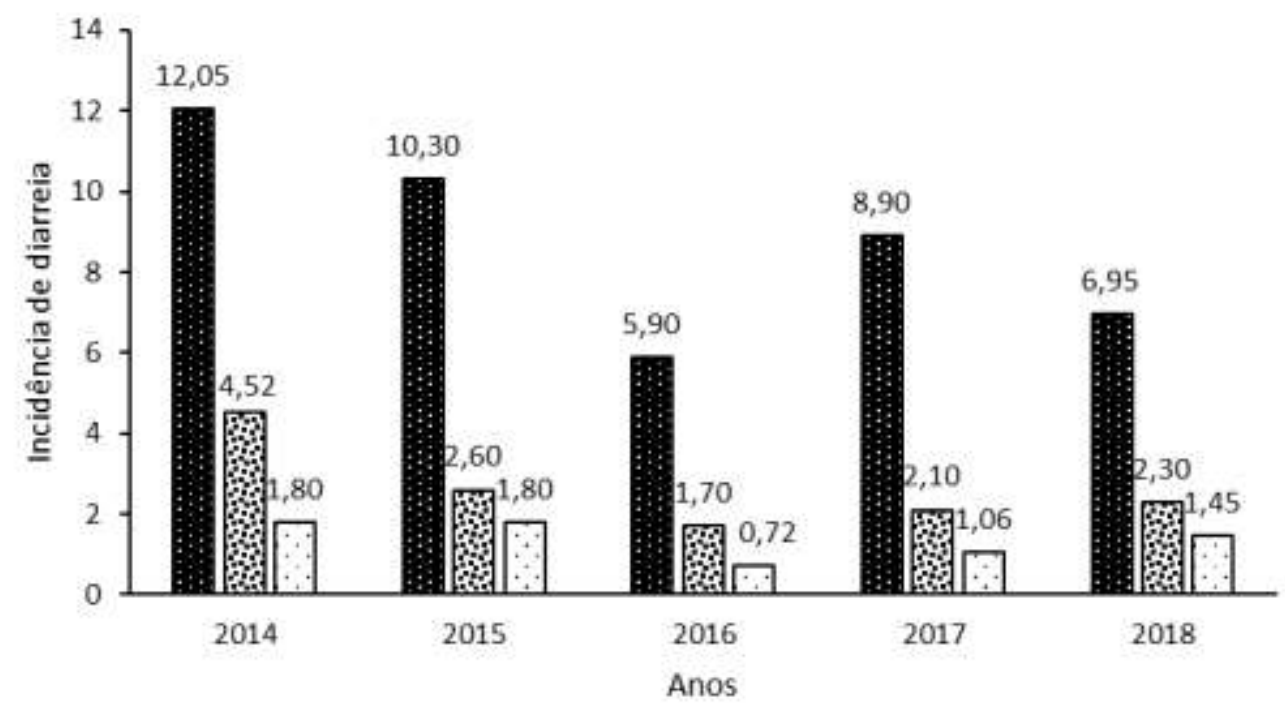

Ma 4 anos E 5 a 9 anos $\square 10$ anos ou mais

Fonte: Autores. Dados disponibilizados pela SIVEP/DDA - Cáceres. Consulta em 19.07.2019

Foram calculadas as incidências de DDAs por faixa etária no município de Cáceres, tendo sido registrados os maiores valores em - todos os anos da série - na faixa situada de 0 a 4 anos: 
Figura 3 - Proporção de Tratamento da Diarreia entre os anos de 2014 e 2018 no município de Cáceres/MT.

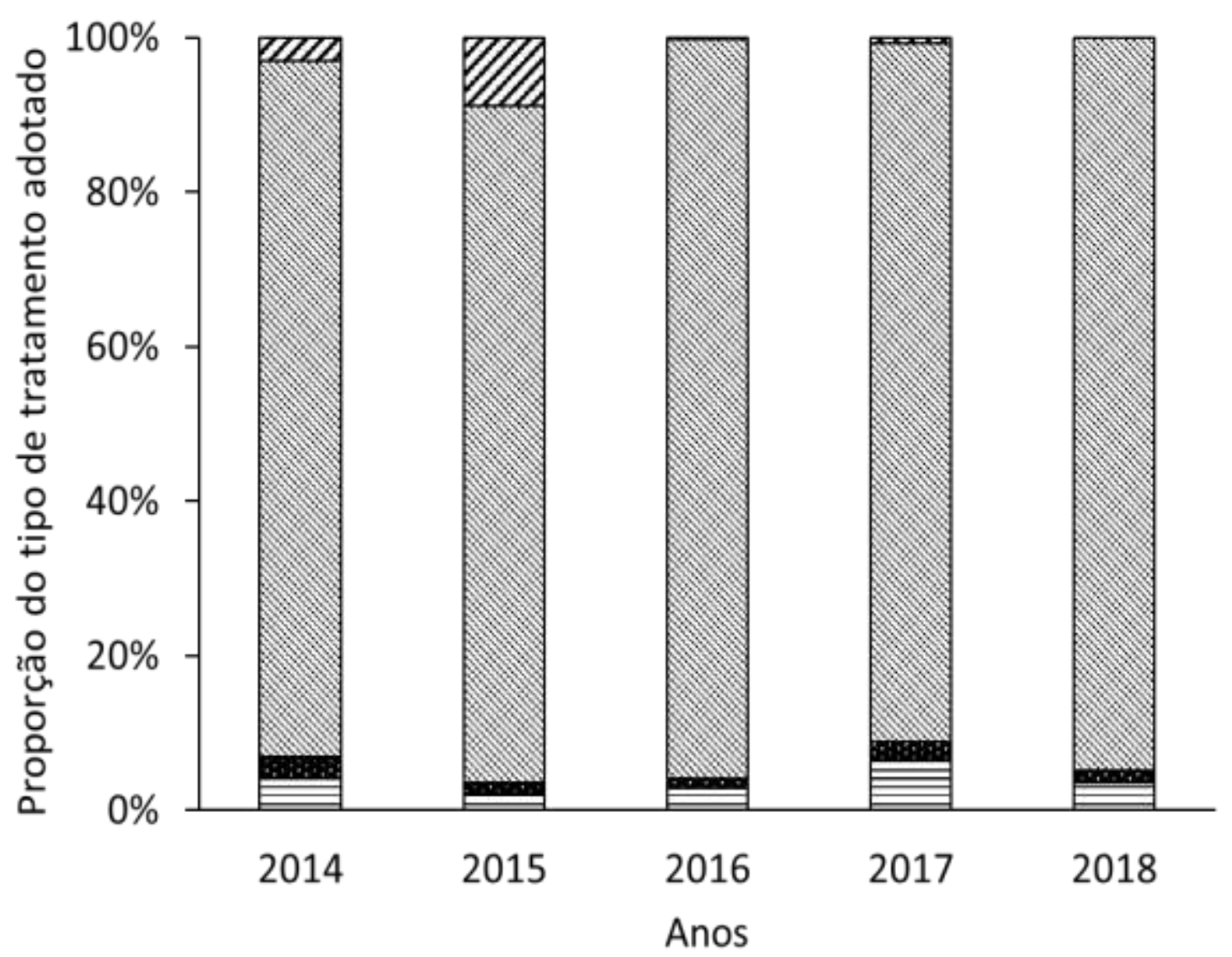

\section{目Plano A Plano B Plano C B Ignorados}

Fonte: Autores. Dados disponibilizados pela SIVEP/DDA - Cáceres. Consulta em 19.07.2019.

A Figura 3 demonstra o percentual de planos de tratamento adotados para as DDAs em Cáceres entre os anos 2014 e 2018. 
Research, Society and Development, v. 10, n. 5, e57910515314, 2021

(CC BY 4.0) | ISSN 2525-3409 | DOI: http://dx.doi.org/10.33448/rsd-v10i5.15314

Figura 4 - DDA notificada por unidade de atendimento em Cáceres/MT no ano de 2018.

\begin{tabular}{|c|c|c|}
\hline \# PSF VILA REAL & n PSF VISTA ALEGRE & $=$ PSF COHAB NOVA \\
\hline$=$ PAM & $=\mathrm{HRC}$ & $=$ PSF DO RODEIO \\
\hline - PSF DO MARAJOARA & - AMBULATÓRIO DA CRIANÇA & - PSF SANTA ISABEL \\
\hline च PSF VILA IRENE & a PSF DO CARAMUJO & - PSF IARDIM PARAÍSO \\
\hline = PSF VITÓRIA RÉGIA & $=$ PSF JD GUANABARA & \\
\hline
\end{tabular}

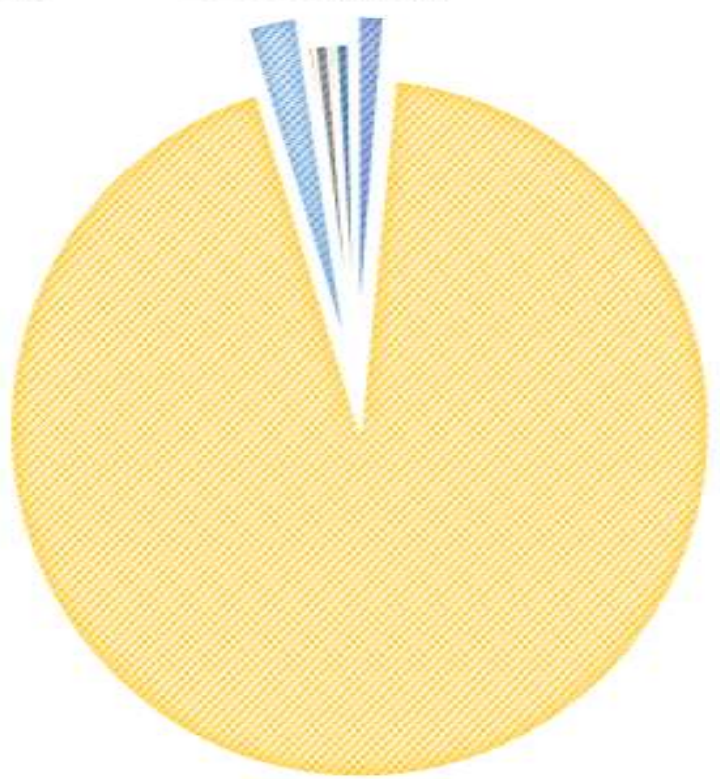

Fonte: Autores. Dados dos formulários impressos arquivados junto a VE Municipal/Cáceres - MT.

Quando os dados são analisados por unidade, verifica-se que o Pronto Socorro Municipal (PAM), seguido do Hospital Regional de Cáceres (HRC) foram responsáveis pelo maior número de notificações em 2018. 
Tabela 3 - Total de semanas de DDA informadas e número de casos em 2018 por Unidade de Atendimento no município de Cáceres/MT

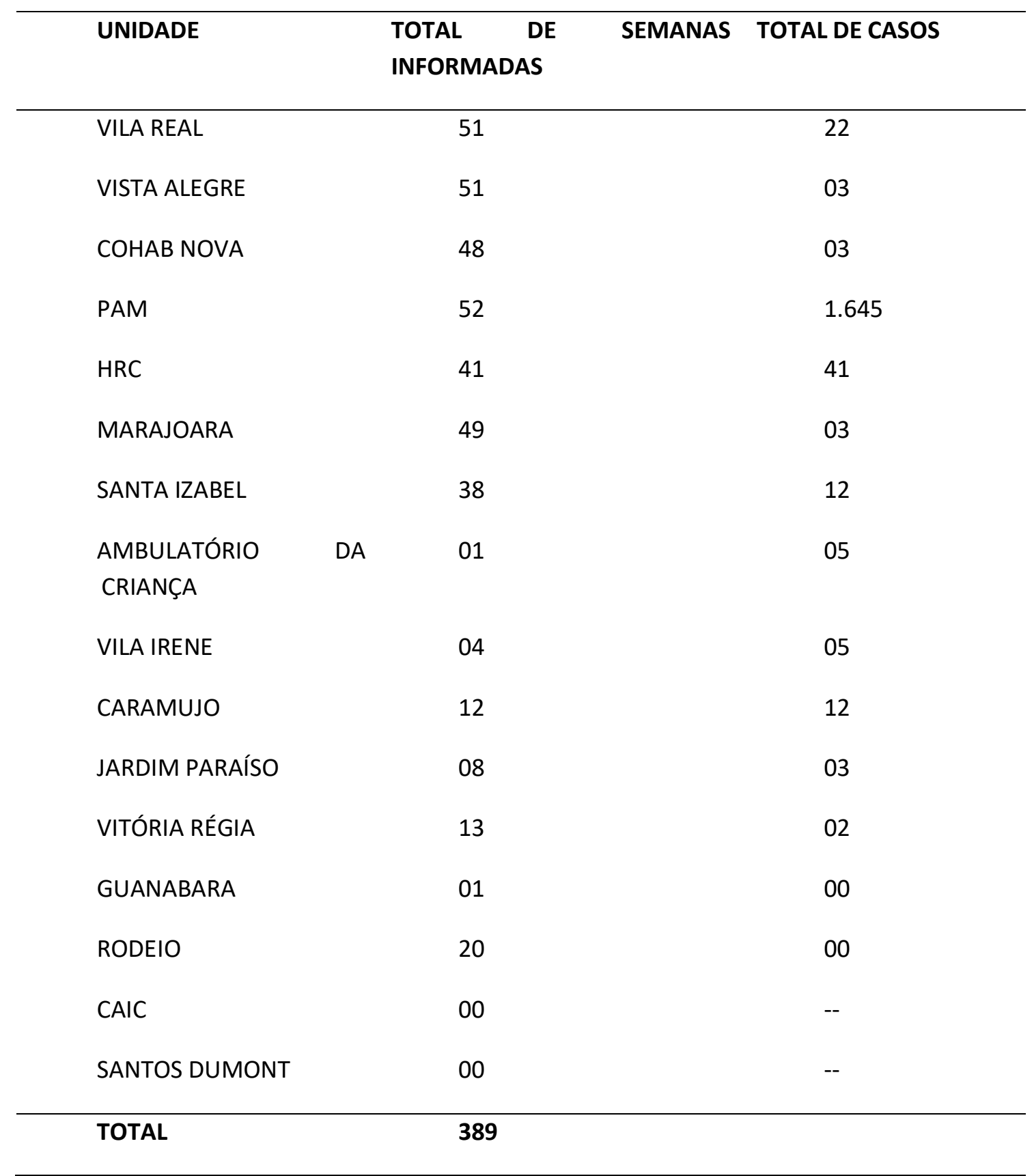

Fonte: Planilhas arquivadas pela VE Municipal.

Na Tabela 3 consta quais e em que quantidade as unidades repassaram informação para a VE municipal no ano de 2018. 
Figura 5 - Diarreias atendidas pelo PAM divididas por localidade em Cáceres/MT, 2018.

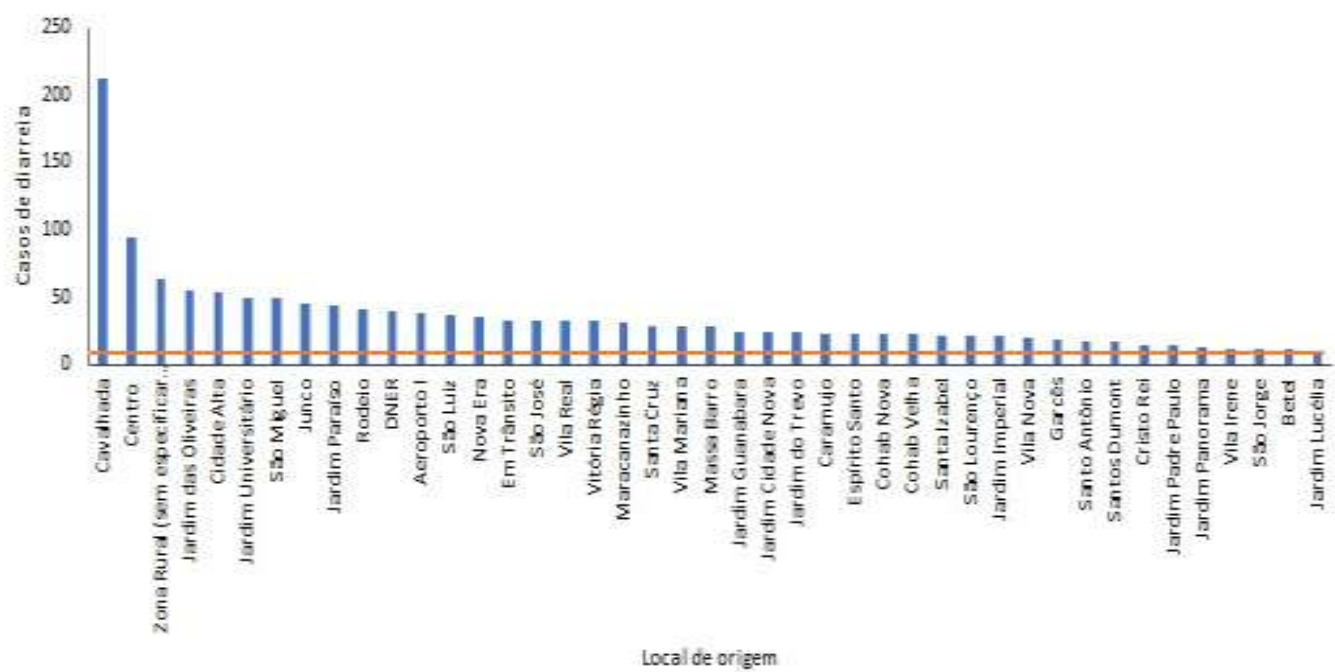

Fonte: Autores. Dados das planilhas arquivadas pela VE Municipal.

Foram elencados nos gráficos as localidades que tiveram, no mínimo, 10 casos no ano (linha laranjada aponta o limite de 10 casos). Quando os casos de diarreia são divididos por local de residência (Figura 5), verifica-se elevado número de casos em bairros sem unidades de saúde da família e na zona rural.

Figura 6 - Pluviosidade, nível do rio e casos de DDA nos anos de 2015, 2017 e 2018 em Cáceres - MT.

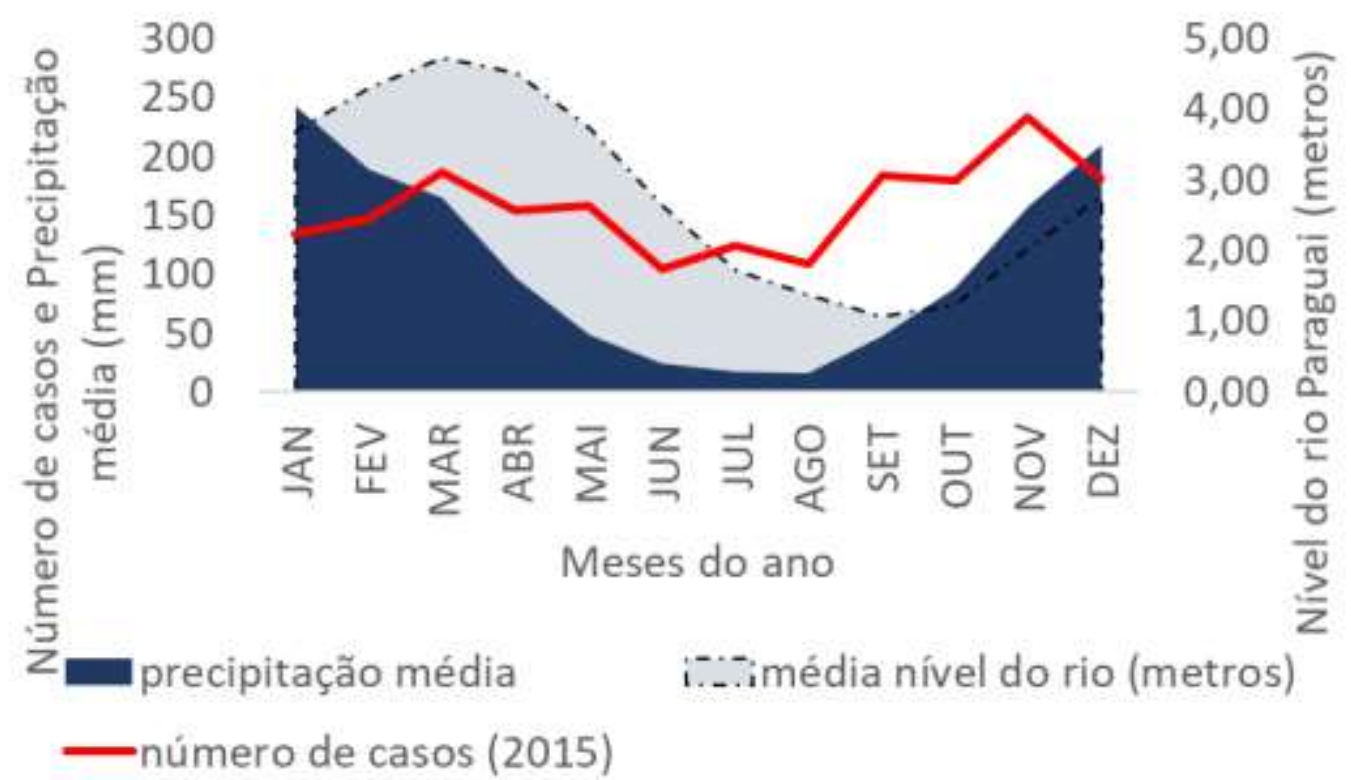

Fonte: Autores. Dados disponibilizados pelo SIVEP DDA, INMET, MARINHA DO BRASIL.

Em relação à sazonalidade, observa-se uma propensão em elevar número de casos. Há uma tendência em aumentar de casos de DDA, quando a quantidade de chuvas é maior ao longo do ano. Essa tendência também acompanha o nível (altura) do 
rio, neste caso específico, o rio Paraguai em Cáceres, utilizado como manancial de abastecimento para aproximadamente 100\% da população residente em área urbana.

\section{Discussão}

Houve redução da incidência de diarreia no município de Cáceres dentro do período analisado. Registra-se, todavia, que quando consultados os dados do SIVEP DDA, estão ausentes de informação 19 semanas epidemiológicas em 2016, o que poderia explicar uma taxa de incidência expressivamente menor para este ano. Para os outros anos, 2014, 2015, 2017 e 2018 , respectivamente, o número de semanas silenciosas foi de 6, 4, 0 e 0. Para efeito de análise, é necessário considerar também, o percentual de unidades repassando informação para a VE municipal ao longo dos anos; neste sentido, constata-se subnotificação das diarreias em Cáceres no período estudado, com maior proporção para os anos de 2016 e 2018. Além disso, quando se analisa as notificações por unidade em 2018, atesta-se que 9 unidades (de 16 possíveis) informaram menos da metade de semanas preconizadas em um ano (52). Entre as quais, duas foram silenciosas o ano todo; duas informaram apenas 01 semana no ano, uma delas o Ambulatório da Criança, cuja atendimento ao público é diversificado (proveniente de diferentes bairros/localidades do município), o que, para esta unidade em especial, talvez se justificasse uma rotina de notificação mais robusta.

A subnotificação de doenças leva a uma descrição equivocada da realidade, impedindo o desencadeamento de ações preventivas com maior agilidade, o que, por sua vez, evitaria novos casos. Em relação as DDAs, redução ou ampliação no número de ocorrências poderia indicar alteração da qualidade da água e/ou necessidade de intensificação de educação em saúde para determinados grupos. Todavia, sem as notificações, o planejamento (bem como a execução) de ações torna-se deficitário. Sobre ações de intervenção adequadas, Brasil (2017) frisa que, "aproximadamente 525 mil crianças menores de 5 anos morrem a cada ano por diarreia. Uma proporção significativa dessas mortes poderia ser evitada se ações em relação à qualidade da água para consumo humano, saneamento e medidas básicas de higiene, como lavagem adequada das mãos, fossem instituídas de forma apropriada".

Acrescenta-se, ainda, que a notificação das DDAs é obrigatória sendo atualmente regulamentada pela Portaria de Consolidação GM/MS n 5, de 28 de setembro de 2017 (Anexo XLIII). Finalmente é importante considerar que, além da subnotificação por parte das unidades públicas, existe aquela ligada aos atendimentos realizados em unidades privadas que, normalmente, não são reportados ao sistema de vigilância municipal, além daquela parcela da população que opta por tratamentos caseiros o que também acarreta em subnotificação (Portela, Leite, Pereira \& Rocha, 2013).

Em que pese a evidência de subnotificações, os dados demonstram maior incidência de DDAs em crianças de 0 a 4 anos. Segundo a WHO (2002) as crianças apresentam maior vulnerabilidade para doenças infecciosas, entre elas, as diarreias. Outros estudos (Façanha \& Pinheiro, 2005, Dias, 2010 e Aguiar, 2016) realizados em outras regiões do Brasil também encontraram maior incidência de diarreia em crianças de 0 a 4 anos. De acordo com os autores, os fatores associados a transmissão de diarreia nessa faixa etária são: precariedade das condições de saneamento, levando ao consumo de água e alimentos com algum grau de contaminação. Segundo Brasil (2017), os menores de 5 anos representaram 15\% das DDAs registradas no Brasil, o que requer atenção do setor saúde, uma vez que, proporcionalmente, esses recortes têm tamanhos de população diferentes e os menores de 5 anos representam apenas 7\% da população total do País.

O manejo adequado das diarreias é a principal estratégia para reduzir a mortalidade por DDA e se compõe de prevenção e tratamento da desidratação. O manual do Ministério da Saúde (Brasil, 2010) preconiza que o paciente portador de DDA seja devidamente avaliado e considerando seu grau de desidratação, o tratamento é definido em Plano A (sem sinais de 
desidratação/tratamento domiciliar), Plano B (com sinais de desidratação/reidratação na unidade e retorno em 24 h/48 h para avaliação) e Plano C (desidratação grave/reidratação venosa e oral na unidade).

Segundo Aguiar (2016), existe uma expectativa por parte do Ministério da Saúde de que a maioria dos casos de diarreia sejam tratados por meio dos planos A e B, pois, além de serem menos onerosos ao sistema de saúde, indicariam menor gravidade das DDAs. Como foi possível constatar nos dados apresentados, as diarreias no município de Cáceres entre os anos de 2014 a 2018 foram tratadas em sua maior proporção, por meio do plano C. Esse resultado contraria aqueles encontrados em outros estudos (Façanha \& Pinheiro, 2005 e Aguiar, 2016). Nesse contexto poderiam ser levantadas algumas hipóteses: 1) os pacientes não procuram a unidade no início da DDA quando os sintomas são mais brandos; 2) as unidades priorizam o registro de ocorrências mais graves; 3) a classificação das diarreias e/ou plano de tratamento foram marcados inadequadamente ou o manejo está inadequado; 4) de fato, no município de Cáceres as diarreias têm apresentado um quadro mais grave e que exigem intervenções mais sistematizadas. A análise das notificações por unidade em 2018 pode explicar, em parte, a maior proporção de tratamentos do tipo $\mathrm{C}$, já que ficou demonstrada uma quantidade de notificações significativamente superior para o Pronto Atendimento Municipal quando comparado com as outras unidades. Por sua natureza (atendimento de urgência e emergência) essa unidade recebe pacientes de todas as localidades e em estado que requer mais atenção/medidas de intervenção. Outras razões pelas quais tem-se recorrido, majoritariamente, ao plano $\mathrm{C}$ para tratamento das DDAs em Cáceres, requerem investigação mais detalhada, incluindo visitas junto às unidades de saúde e até mesmo verificações em prontuários de pacientes atendidos no período do estudo.

Quando são analisados os dados de notificação de DDA por localidade constata-se maior quantidade para bairros populosos, quais sejam Cavalhada e Centro, bem como para àqueles não contemplados por estratégias de saúde da família (Cavalhada, Centro, Cidade Alta, São Miguel, DNER). Ademais, interessante chamar a atenção para os casos registrados nos bairros Jardim das Oliveiras e Jardim Paraíso, por exemplo, que somaram juntos 99 casos de DDA em 2018 (atendidos no PAM) e possuem ambos como referência a Unidade Jardim Paraíso. Se o conjunto de notificações é analisado por unidade, no entanto, verifica-se que a unidade Jardim Paraíso notificou apenas 3 casos no ano e repassou informação em somente 08 semanas num total de 52 preconizadas. A mesma inferência pode-se fazer para a Unidade Jardim Guanabara que repassou informação em 01 semana negativa em 2018; mas no consolidado de atendimento pelo PAM há 24 pacientes que declaram residir nesse bairro. A unidade Vitória Régia contempla os bairros Vitória Régia, Santo Antônio, Jardim Popular e Jardim Primavera (Barelli, 2012); com 54 registros de atendimento de DDA em 2018 no PAM, foram notificados pela Unidade Vitória Régia no mesmo período 02 casos da doença. Ocorrência semelhante está para a Unidade do Santos Dumont que em 2018 não repassou informação sobre DDAs eventualmente atendidas na unidade, porém, quando recorre-se aos registros do PAM por localidade, verifica-se que 17 pacientes têm como local de residência o bairro Santos Dumont; se somados os casos provenientes do Jardim Universitário (49 no total) o número de DDA ocorrida em território da unidade Santos Dumont é elevado para 66.

Também se registra elevado número de atendimentos de usuários cuja origem é a Zona Rural (fazendas, sítios e assentamentos); na Zona Rural, as fontes de abastecimento de água são normalmente constituídas de poços nos quais a água não passa por nenhum tipo de tratamento. Resultados encontrados por Nodari, Neves, Silva \& Nodari (2018) em estudo realizado em assentamentos do município de Cáceres corroboram tal afirmação, uma vez que aproximadamente $70 \%$ dos moradores entrevistados na pesquisa responderam consumir água natural e sem tratamento prévio. Ademais, Valentin, Diniz, Campos, Melo, Esquerdo \& Correia (2020) também verificaram alta incidência de doenças diarreicas em população de ribeirinhos do Pará, cujo consumo era de água não tratada.

Estudos realizados por Buhler \& Souza (2011), apontam que as maiores concentrações de chuva na região de Cáceres ocorrem, normalmente, nos meses de janeiro, fevereiro, março e dezembro; o período de águas altas (maiores variações no 
nível do rio) correspondem ao período de maior precipitação (primeiro trimestre). Lázaro \& Oliveira Junior (2020) reforçam que no Pantanal Norte (região de Cáceres) os períodos de cheia/seca - pulso de inundação - são fortemente influenciados pela pluviosidade. Na série histórica analisada pelo estudo (2015, 2017 e 2018) verifica-se que o número de casos de diarreia tende a subir nos meses em ocorrem as maiores quantidades de chuva e de maior nível do rio; essa tendência pode ser explicada em parte porque as chuvas aumentam a presença de coliformes nas águas dos mananciais, devido ao arraste de excretas humanas e animais, o que favorece a contaminação da água não tratada e também em águas tratadas, caso as rotinas, estruturas e/ou equipamentos dos sistemas de abastecimento não estiverem condizentes com os padrões exigidos. Fonseca (2018) em estudo realizado em cidades da Amazônia Ocidental explicou que valores altos de DDA no período de águas altas, ocorre por que o rio pode invadir áreas antropizadas, como ruas e casas, misturando-se às aguas servidas. Outros estudos, tais como como àqueles realizados por Albuquerque, Pessoa, Gomes \& Santana (2020) no estado do Maranhão também encontraram maior número de casos de diarreias no período chuvoso nas aldeias indígenas daquele estado.

\section{Considerações Finais}

À vigilância em saúde compete: melhorar a qualidade/quantidade de notificações de DDA em unidades de atenção básica, de modo que seja possível acompanhar mais proximamente o panorama das diarreias no município de Cáceres: se são casos graves, se a maioria em crianças, se relacionados à água; essa melhoria depende de os profissionais estarem capacitados e/ou sensibilizados para o registro das informações. Sendo assim, seria importante que os responsáveis pela vigilância em saúde municipal realizassem reuniões técnicas e/ou capacitações sobre as DDAs (diagnóstico, manejo, monitorização, vigilância) voltadas aos profissionais das unidades.

Incluir setores (vigilância sanitária, secretaria de ação social, departamento de abastecimento de água) em ações que visem a melhoria do quadro de saúde da população quando o tema for a ocorrência de DDA, no entanto, para que participem, necessitam receber orientações/dados por parte da vigilância epidemiológica, para que intervenham de diferentes maneiras: vigilância sanitária em vistoria das condições dos sistemas de abastecimento de água (públicos e privados), secretaria de ação social em levantamento sobre as condições de vida das famílias e o departamento de água corrigindo os problemas encontrados na qualidade da água, bem como ampliando a distribuição de água potável para toda a população.

À secretaria municipal de saúde compete: ampliar a cobertura de atenção básica para garantir o acesso da população em ações de prevenção e assistência sem a necessidade recorrer aos serviços de emergência.

Estudos futuros sobre o comportamento das diarreias podem incluir dados sobre a água consumida pela comunidade local, de modo que a relação entre as doenças diarreicas e a qualidade hídrica fique mais seguramente estabelecida e forneça subsídios ao poder público para intervenções mais precisas e que melhorem as condições de saúde da população.

\section{Agradecimentos}

Agradecemos a Vigilância Epidemiológica Municipal de Cáceres pelo empréstimo das planilhas de diarreia do ano de 2018 para análise, à direção do Escritório Regional de Saúde de Cáceres por oportunizar participação no EPISUS Fundamental, gerando a publicação deste trabalho; à SES/MT e ao Ministério da Saúde pelo oferecimento do curso.

\section{Referências}

Aguiar, D. B. (2016). O perfil epidemiológico da diarreia aguda e sua correlação com indicadores ambientais e sócio-econômicos do município de Boa VistaRoraima. Dissertação (Mestrado) - Universidade Federal de Roraima.

Agência Nacional das Águas. (2010). https://www.ana.gov.br/as-12-regioes-hidrograficas-brasileiras/paraguai 
Albuquerque, D. F., Pessoa, F. C. L., Gomes, E. P. \& Santana, L. R. (2020). Relação entre o abastecimento de água e a ocorrência de doenças em indígenas no estado do Maranhão, Brasil. Research, Society and Development.

Barelli, C. S. G. A. P., Galbiati, C., Scochi, M. J., Silva, A, R. B. \& Demarchi, F. F. (2012). Ocorrência de diarreia infantil e qualidade da água de consumo doméstico em Cáceres/MT, município do Pantanal Brasileiro. Seminário Nacional de Diretrizes da Enfermagem na Atenção Básica em Saúde.

Brasil. (2010). Ministério da Saúde. Capacitação em monitorização das doenças diarréicas agudas - MDDA: manual do monitor.

Brasil. (2018). Ministério da Saúde. Saúde Brasil 2017: Uma análise da situação de saúde e os desafios para o alcance dos Objetivos de Desenvolvimento Sustentável. Brasília, DF.

Buhler, H. F., Neves, S. M. A. S., Ignotti, E. \& Souza, C. K. J. (2012). Indicadores integrados de saúde e ambiente para morbimortalidade por diarreia infantil nos Estados de Mato Grosso e Mato Grosso do Sul, com ênfase na bacia do alto Paraguai. Anais $4^{\circ}$ Simpósio de Geotecnologias no Pantanal.

Buhler, B. F., Souza, C. A., \& Oliveira Junior, E. S. (2013). Qualidade da Água do Rio Paraguai no Perímetro Urbano em Cáceres - MT, Brasil. Revista Geopantanal. UFMS, Grupo de Pesquisa Pantanal Vivo.

Dias, D. M. (2010). Morbimortalidade por gastroenterites no Estado do Pará. Revista Pan Amazônica Saúde.

Façanha, M. C. \& Pinheiro, A. C. (2005). Comportamento das doenças diarreicas agudas em serviços de saúde de Fortaleza, Ceará, Brasil, entre 1996 e 2001. Caderno de Saúde Pública.

FNP - Frente Nacional dos Prefeitos. (2018). G100: municípios populosos com baixa receita per capita e alta vulnerabilidade sócio econômica.

Fonseca, P. M. A. (2018). Influência de variáveis hidro-climáticas na ocorrência de diarreias em menores de 5 anos na Amazônia Ocidental. (Tese) Doutorado. Instituto Nacional de Pesquisas da Amazônia.

Gil, A. C. (2002). Como Elaborar Projetos de Pesquisa? Atlas.

Instituto Brasileiro de Geografia e Estatística. (2010). http://www.ibge.gov.br/cidades.

Instituto Brasileiro de Geografia e Estatística. (2019). https://agenciadenoticias.ibge.gov.br/agencia-detalhe-de-midia.

Instituto Nacional de Metereologia. http://www.inmet.gov.br/portal/index.php?r=bdmep/bdmep.

Lázaro, W. L., Oliveira Junior, E. S., Da Silva, C. J. \& Muniz, C. C. (2020). Climate change reflected in one of the largest wetlands in the world: an overview of the Northern Pantanal water regime. Acta Limnologica Brasiliensia.

Mato Grosso (2007). Censo Econômico dos Municípios. http://www.indicador.seplan.mt.gov.br/censo

Marinha do Brasil (2019). Serviço de Sinalização Náutica do Oeste. https://www.marinha.mil.br/ssn-6/?q=alturaAnterioresRios.

Nodari, P. R. G., Neves, S. M. A. S., Silva, G. J. O. \& Nodari, D. E. (2018). Indicadores de saúde ambiental nos assentamentos do município de Cáceres faixa fronteiriça Brasil/Bolívia. Journal Health NPEPS.

Oliveira-Junior, E. S., Buhler, B. F., Muniz, C. C. \& Furlan, A. O. (2014). Córregos urbanos do município de Cáceres-MT, Brasil: Um Olhar para a conservação. Revista Eletrônica em Gestão, Educação e Tecnologia Ambiental.

Oliveira Junior, E. S. (2021). Aplicação de protocolos de avaliação rápida como ferramenta robusta na qualificação ambiental em dois córregos urbanos que deságuam no Rio Paraguai. Raega - O Espaço Geográfico em Análise.

Papini, S. (2009). Vigilância em Saúde Ambiental - Uma Nova Área da Ecologia. (2a ed.), revista e ampliada. Química Nova.

Portela, R. A., Leite, V. D., Pereira, C. F. \& Rocha, E. M. F. M. (2013). Comportamento das doenças diarreicas nas mudanças sazonais no município de Campina Grande - PB. HYGEIA/Revista Brasileira de Geografia Médica e da Saúde.

São Paulo (2008). Monitorização das Doenças Diarreicas Agudas. Normas e Instruções.

Souza, C. O., Silva, M. C. M., Loureiro, E. C. B., Sousa, E. B., Ramos, E. M. L. S \& Souza, V. F. M. (2012). Fatores de risco e etiologia infecciosa da doença diarreica no Município de Juruti, Estado do Pará, Brasil. Revista Pan Amazônica de Saúde.

Valentim, L. A., Diniz, D. W. R., Campos, G. M. P., Esquerdo, G. A. M., Melo, G. C. C. \& Correia, Y. P. B. (2020). Vulnerabilidades individuais e sociais para ocorrência de parasitoses em comunidades ribeirinhas da Amazônia Paraense. Research, Society and Development.

Instituto Trata Brasil. (2011). A importância do Saneamento Básico para a Saúde nos 25 municípios do Pacto em Defesa das Cabeceiras do Pantanal - Uma Aliança para o Desenvolvimento Sustentável da Região. http://www.tratabrasil.org.br/images/projetos-sociais/cabeceiras-pantanal/estudo-pantanal-online.pdf 